\title{
LE RÔLE DES PROTÉINES COMME FACTEURS D'APPÉTENCE POUR LES GALORIES CHEZ LE POULET EN CROISSANCE
}

\author{
C. CALET et Françoise BARATOU \\ Station de Recherches avicoles, \\ Centre national de Recherches zootechniques, Fouy-en-Fosas (Seine-et-Oise).
}

Au cours de l'année 1961-1962 nous nous sommes intéressés à l'étude de l'efficacité azotée selon le mode de distribution des aliments. Ceci nous a amené à observer que les protides manifestent une action sur la croissance qui est la résultante de plusieurs facteurs que nous avons cherché à analyser. En particulier, nous nous sommes attachés récemment à l'étude du rôle des matières azotées sur l'appétit.

Un premier travail concerne l'évolution du coefficient d'efficacité protidique de deux sources azotées en fonction d'une part de la quantité d'azote ingérée et d'autre part du mode de rationnement. Le schéma du protocole expérimental est le suivant : Les matières azotées étudiées sont dans une première expérience la farine de poisson et le tourteau d'arachide qui ont été choisis en raison de la divergence de leur valeur biologique. Chacune d'entre elles entre dans la composition d'une ration protidique renfermant $50 \%$ de matières azotées totales $(\mathrm{N} \times 6,25)$ et comprenant les vitamines nécessaires à l'animal. On constitue par ailleurs, une ration protéiprive à partir de glucose, amidon, huile de mais et minéraux. Une partie des lots reçoit l'une et l'autre de ces rations dans des mangeoires séparées. L'autre partie des animaux reçoit une seule ration complète, préparée en mélangeant les deux rations. Tous les animaux reçoivent journellement des quantités limitées de matières azotées totales (de $2 \mathrm{~g}$ à $15 \mathrm{~g}$ par jour et par animal). Ainsi les lots recevant une ration complète disposent au total d'une quantité restreinte d'aliment. Chez les animaux nourris des deux rations, seule la ration protidique est restreinte alors que la ration protéiprive est distribuée à volonté. Nous avons précédemment observé (Calet, Jouandet, Baratou, 1961) que le poussin est capable de consommer spontanément une quantité convenable d'aliment protéiprive afin d'assurer son besoin énergétique.

Le graphique 1 illustre nos résultats. Lorsque la ration est complète (courbes de croissance 1 et 2 ) nous retrouvons les conclusions établies depuis 1930 par Heuser lorsqu'il recherchait le besoin azoté du poussin : au fur et à mesure que la quantité d'azote ingéré augmente la croissance est améliorée puis passe par un maximum et enfin décroît. Il convient toutefois de souligner que ce besoin azoté défini dans nos conditions expérimentales dépend de la nature des matières azotées. Il est plus faible lorsque s'accroît la valeur biologique de la matière azotée.

Lorsque le poussin reçoit simultanément deux rations et qu'il dispose à volonté des calories non azotées, la croissance est constamment ascendante au fur et à mesure que le taux protidique du régime est plus élevé. Dans ces conditions, il est impossible de définir une croissance optimum en fonction de la quantité d'azote ingérée. 


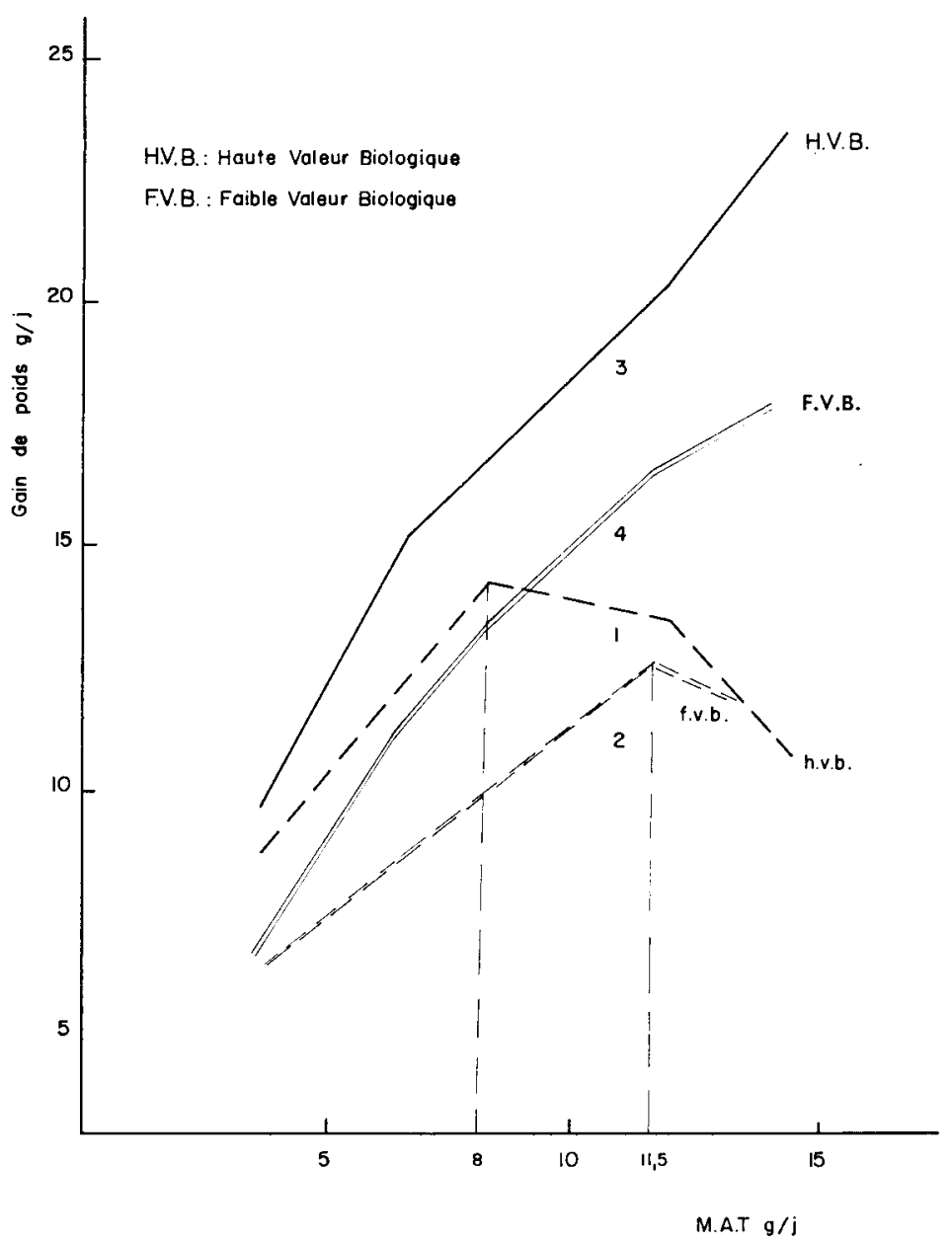

Fig. $I$.

Nous avons précédemment établi que la consommation spontanée par gramme de protide est d'autant plus forte que les matières azotées possèdent une valeur biologique plus élevée. Ceci est vrai pour une quantité de matière azotée donnée $(5,1 \mathrm{~g}$ par poussin et par jour). Nous avons recherché comment cette consommation spontanée varie en fonction du niveau alimentaire d'azote. Le graphique 2 fournit les valeurs du coefficient d'efficacité protidique en fonction du rapport entre les calories volontairement consommées et les matières azotées. Dans l'un et l'autre cas les coefficients d'efficacité protidique passe par un optimum mais cet optimum correspond à des rapports $\mathrm{C} / \mathrm{P}$ beaucoup plus élevés lorsque la valeur biologique des protides est plus forte.

On peut adresser une critique à notre expérimentation. La consommation spontanée d'énergie engendrée par les matières azotées peut ne pas être forcément le fruit de la valeur biologique des protides sensu stricto mais être la conséquence de principes non protidiques accompagnant les matières azotées. Nous nous sommes adressés en effet à des aliments naturels et non pas à des protéines purifiées. Les expériences qui 
vont être rapportées ont tenté de vérifier dans quelle mesure la valeur biologique des protides est responsable de la consommation d'énergie.

Tout d'abord pour éviter la critique par laquelle les aliments naturels azotés fournissent à l'animal d'éventuels " facteurs d'appétence », nous avons repris dans une seconde expérience le même protocole expérimental que dans la première expérience en modifiant l'origine des matières azotées. Cette fois nous avons fait varier l'efficacité des protides non plus en nous adressant à deux sources distinctes mais en constituant des

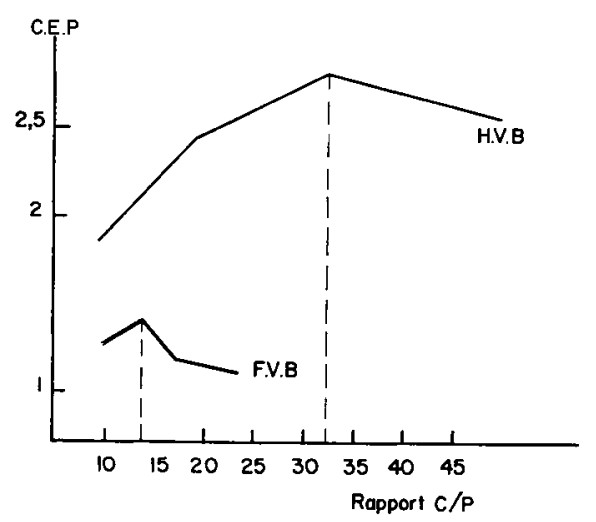

Fig. 2.

mélanges de deux matières azotées en proportion différente. Il s'agit du gluten de blé et de la farine de poisson. Un des aliments protidiques renferme $80 \%$ des matières azotées sous forme de farine de poisson et $20 \%$ sous forme de gluten. Dans l'autre aliment protidique les proportions sont inversées (protides de gluten $80 \%$ et protides de la farine de poisson $20 \%$ ).

Nous ne donnerons pas de résultats chiffrés car les conclusions du deuxième essai sont rigoureusement superposables à celles du premier.

En dépit de ce résultat positif, nous ne pouvions pas affirmer que la valeur biologique des protides est responsable à elle seule de la consommation d'énergie. Cela ne peut être vrai que dans la mesure où des aliments azotés d'origine différente et possédant la même efficacité protidique conduisent à la même consommation spontanée d'énergie. Aussi, dans une troisième expérience nous avons tenté de constituer des régimes protidiques possédant la même valeur biologique en mélangeant des aliments azotés naturels dans des proportions variables (4 mélanges à base de tourteau d'arachide et de farine de poisson, 4 autres mélanges à base de tourteau de sésame et de caséine). Nous avons alors confronté les coefficients d'efficacité protidique de ces régimes et les consommations spontanées d'énergie qu'ils engendrent avec les résultats fournis par le tourteau de soja.

Le tableau 1 donne pour les différents régimes les valeurs relatives du coefficient d'efficacité protidique et de la consommation d'aliment non azoté lorsque l'on attribue au soja la valeur 100 .

Apparemment il semble que l'on puisse établir une concordance parfaite entre efficacité azotée et consommation spontanée d'énergie indépendante de l'origine des matières azotées. Mieux, pour les régimes dont les efficacités sont les plus proches, 
TABLEAU 1

Classement des matières azotées d'après leur C. E. P. et la consommation d'aliment non-azoté qu'elles provoquent.

\begin{tabular}{|c|c|c|c|c|c|}
\hline Lots & $\begin{array}{r}\text { Répartition des } \\
\text { des différents ali } \\
\text { sur la bas }\end{array}$ & $\begin{array}{l}\text { mati } \\
\text { ments } \\
\text { de } 1\end{array}$ & $\begin{array}{l}\text { eres azotées } \\
\text { protidiques } \\
\text { 'azote }\end{array}$ & $\begin{array}{c}\text { Coefficient d'efficacité } \\
\text { protidique }\end{array}$ & $\begin{array}{c}\text { Consommation } \\
\text { d'aliment } \\
\text { non-azoté }\end{array}$ \\
\hline V & $\frac{\text { Sésame }}{\text { Caseine }}$ & $=$ & 3,0 & 142,7 & 132,5 \\
\hline I & $\frac{\text { Arachide }}{\text { Poisson }}$ & $=$ & 1,8 & 101,1 & 102,9 \\
\hline $\mathrm{S}$ & Soja & & & 100,0 & 100,0 \\
\hline II & $\frac{\text { Arachide }}{\text { Poisson }}$ & $=$ & 2,2 & 99,4 & 94,6 \\
\hline III & $\frac{\text { Arachide }}{\text { Poisson }}$ & $==$ & 2,6 & 96,7 & 90,2 \\
\hline IV & $\frac{\text { Arachide }}{\text { Poisson }}$ & $=$ & 3,0 & 92,2 & 86,2 \\
\hline VI & $\frac{\text { Sésame }}{\text { Caséine }}$ & & 20,0 & 84,4 & 89,9 \\
\hline VII & $\frac{\text { Sésame }}{\text { Caséine }}$ & $=$ & 26,6 & 79,7 & 82,0 \\
\hline VIII & $\frac{\text { Sésame }}{\text { Caséine }}$ & $=$ & 32,0 & 76,9 & 80,5 \\
\hline
\end{tabular}

c'est-à-dire pour le régime soja (lot $\mathrm{S}$ ) et pour les régimes arachide-poisson (lots I et II), on pourrait presque confondre les valeurs des coefficients d'efficacité protidique et des consommations d'énergie. Les valeurs fournies dans le cas des régimes sésamecaséine sont plus éloignées de celles du soja mais une même proportionnalité demeure entre les deux séries de mesures.

La concordance entre les deux séries de mesure n'est malheureusement qu'apparente. En effet pour chacun des types de régime protidique considéré, nous avons calculé le coefficient de corrélation entre efficacité azotée et consommation d'énergie. Ce coefficient est extrêmement élevé pour les régimes soja et sésame-caséine puisque sa valeur est de 0,81 . En revanche la valeur du coefficient n'est que de 0,68 pour les régimes arachide-poisson. Ainsi il apparaît une relation extrêmement étroite entre l'efficacité azotée d'un protide et son aptitude à développer l'appétit calorique du poussin toutes les fois que les matières azotées sont d'origine végétale ou sont des protides purifiés. Par contre, dans le cas des aliments d'origine animale (farine de poisson) la seule valeur biologique de leurs protides n'est pas en mesure d'expliquer la totalité de leurs effets sur l'appétit. Nous avons des raisons de penser que la vitamine $\mathrm{B}^{12}$ qui accompagne les farines d'origine animale est responsable d'une partie des effets supplémentaires sur la consommation d'aliment et qui ne sont pas contrôlés par les protides. 